\title{
Sizing and Actuation of Child Lower Limb Exoskeleton
}

\author{
Mohamad Kadrofaki, Moustafa A. Fouz, Khaled Fouda, Samer Alfayad, and Eric Dychus
}

\begin{abstract}
In this article, the sizing problematic has been treated for a newly developed exoskeleton to operate effectively. The objective of the latter exoskeleton is to aid the paralyzed children to move their lower extremities and perform a complete gait motion and other physical activities. The applied torques on each actuator's are calculated by defining the quasistatic loads generated during the worst-case configurations of the children lower limbs, and also, using a normalized biomechanical data extracted from literature. Also, a virtual model is developed using Matlab-Simulink to verify the results.
\end{abstract}

Index Terms-Exoskeleton, child, lower limb, actuator sizing.

\section{INTRODUCTION}

Over 68 million people worldwide have lower limb paralysis due to several causes, and the same result is chasing them. The traditional solution for mobility problem is to use wheel chair. Hence, using wheel chairs may solve a part of the problem, but it still limits their freedom. As well as sitting on a chair for a long time may causes some biological disorders such as back and legs ulcers, muscular atrophy, Osteoarthritis, ... etc. Also there are some psychological drawbacks affecting them due to their feel of losing the ability of doing usual vital daily movements, and their dependency on others to get help. The feeling of disability may aggravate the mood of depression and rescue their desire to live. Where those people should still have the right to stand up, walk, feel the independence, work, and have their natural life just like the others.

Thanks to exoskeletons, many patients are able to move on their legs, they can stand up and walk, and maybe climb stairs also. But the main exoskeletons on the market are not customized for children needs. Where the needs of children, and their requirements in their exoskeletons, differs from adults or elderly people. As their bodies have smaller sizes, weights, and have a much lower ability to wear complicated exoskeletons, it's improper for a child to wear an exoskeleton with large motor size for example. Also the risk of injury while using a powerful exoskeleton is really high, especially they have special mindset in dealing with their stuff. And the tendency of trying and exploring an unsecured, powerful wearable device can cause harmful effects and the possibility of injury may occurs.

Thus, the exoskeleton will show a practical solution to the children patients, but the actuation sizing of this solution is

Manuscript received on May 6, 2018; revised on July 19, 2018.

The authors are with UVSQ, Paris-Saclay University 10-12 Avenue de l'Europe, 78140 Vélizy, France (e-mail: kardmoha@lisv.uvsq.fr, moustafa.fouz@lisv.uvsq.fr, samer.alfayad@lisv.uvsq.fr, eric.dychus@sandyc.fr). still an open question because it depends on several conditions, among these conditions we note the inertial parameters such as (the weight, size, length), the kinematic and dynamic parameters such as (the walking speed, wearer weight, type of the activity to do, level of performance...etc.), and other medical constraints such the type of the disorder or the injury... etc.

The paper is organized as follows: In Section II, a literature is surveyed on current exoskeletons. In Section III, a biological study is illustrated to get better understanding for the human lower limb abilities and gait analysis. Then, in Section IV the actuator sizing techniques shown. In Section V the calculation results are illustrated and summed up. calculations and verification are shown in Section VI. Finally, the conclusion is presented at the end.

\section{LITERATURE REVIEW}

Different functional compensation and/or rehabilitation exoskeletons were presented in the last years. The most notable are the Hybrid Assistive Leg (HAL), developed by Cybernics Laboratory of the University of Tsukuba in Japan [1], the Rewalk walking assitstance exoskeleton, designed by Argo Medical Technologies [2]. The EKSO developed by EKSO Bionics [3]. Indego developed in the University of Vanderbilt (Tennessee, United States) [4]. The phoenix exoskeleton developed by SuitX [5]. The Berkeley Lower Extremity Exoskeleton (BLEEX) [6], developed by the University of Berkeley in the USA.

The power assist device HAL is a walking aid system which is also being used for people with walking disorders, only hip, knee joints, and ankle are actuated in the sagittal plane, the other degrees of freedom in transverse and frontal planes are not actuated. It successfully walks and carries its own power supply and has been designed to assist the wearer's muscles by measuring users own muscle activity. HAL has a height of 1.6 meters and weights $23 \mathrm{~kg}$; the battery allows for $160 \mathrm{~min}$ of continuous operation and enables the exoskeleton to lift up to $70 \mathrm{~kg}$. It is divided between the top part and the two mobile parts of the bottom. Top section weight is around $8 \mathrm{~kg}$ and the parts attached to legs together weigh $15 \mathrm{~kg}$. Human operators attach to HAL at the waist with a belt, and at the calf and thigh using harnesses. The HAL device doesn't transfer the forces generated by the weights and the payload to the ground, but it augments the joint torques at the level of hip, knee, and ankle.

Rewalk system offers the ability to patients having lower limbs disabilities to stand, sit, walk, and ascend / descend stairs.

The hip and knee are actuated in the sagittal plane of the human body by a series of DC motors, so the ankle is 
passive joint and having a pre-defined range of motion and spring for dorsi-flexion movement. It can reach a maximum velocity of walking of $0.6 \mathrm{~m} /$ second $(2.2 \mathrm{~km} /$ hour $)$ [7]. It owns the use of crutches to address stability of the whole system during motion. A sensory system located on the upper body measures its inclination in order to recognize the user's will to generate movement.

\begin{tabular}{lccc} 
& TABLE I: SPECIFICATIONS OF BLEEX JOINTS \\
\hline & $\begin{array}{c}\text { BLEXX } \\
\text { ROM }\end{array}$ & $\begin{array}{l}\text { Human } \\
\text { torque }\end{array}$ & Bleex Max torque \\
\hline $\begin{array}{l}\text { Ankle flexion } \\
\text { lextension }\end{array}$ & $\pm 45^{\circ}$ & -120 N.m & $-200 / 155$ N.m \\
\hline Knee flexion & $121^{\circ}$ & $-35 / 60$ N.m & $-100 / 140$ N.m \\
\hline $\begin{array}{l}\text { Hip flexion } \\
\text { extension }\end{array}$ & $\pm 121^{\circ} / 10^{\circ}$ & $-80 / 60$ N.m & $-150 / 130$ N.m \\
\hline
\end{tabular}

Ekso exoskeleton system similar to Rewalk, based on actuation of the Hip and knee by a DC motors, and the user requires crutches to maintain stability. It weighs $20 \mathrm{~kg}$ and has a maximum walking speed of $3.2 \mathrm{~km} / \mathrm{h}$, while its autonomy is 3-6 hours. Also, it can execute sit-to-stand and stand-to-sit operations and walk in a straight line.

Indego or Vanderbilt exoskeleton is an active orthotics for rehabilitation use it weighs about $12 \mathrm{~kg}$, and it has only the hip and knee joints as actuated degrees of freedom. The ankle joint in sagittal plane is not presented. The joints are powered by DC motors through a gear reducer. So the actuated joints are provided by a 12 N.m continuous torque. A power supply, made from lithium polymer, achieves a one hour of autonomy. The device can support people weighting up to $91 \mathrm{~kg}$.

The phoenix exoskeleton active orthotics system made by university of California, has an objective to regenerate the motion of the disabled persons. This exoskeleton is lightly adjustable and lightweight $(12 \mathrm{Kg})$, the accumulators can achieve an autonomy of 4-8 hours, the users can have a height between $160 \mathrm{~cm}$ and $187 \mathrm{~cm}$ only. while the wearer and the device weights of should not exceed $91 \mathrm{Kg}$, and to maintain stability the user must use crutches. A significant flexion contractures lightly limited to $35^{\circ}$ at the hip and $20^{\circ}$ at the knee.

BLEEX is a robotic exoskeleton for human performance augmentation capable of carrying its own weight plus an external payload [8]. It's energetically autonomous and walks at the average speed of $1.3 \mathrm{~m} / \mathrm{s}$ while carrying a $34 \mathrm{~kg}$ payload due to the generated hydraulic power [9]. This exoskeleton has 7 DoF driven with hydraulic cylinders: 3 at the hip, 1 at the knee and 3 at the ankle. The associated ranges of motion (ROM) of each joint are illustrated in table Iref $\{$ table:Bleex_table $\}$

Joint angles, torque, and power requirements are determined from human motion analysis based on a $75-\mathrm{kg}$ human walking on flat ground at roughly $1.3 \mathrm{~m} / \mathrm{s}$. During design, joint motion was intended to be slightly less than the maximum human range of motion for safety; however, some joint ranges had to be reduced to avoid (mechanical) singularities.

Hence, to regenerate the feeling of independence, an exoskeleton that can able to regenerate the walking and all the other activities that children need to do is still an open question on this level. The state of the art at the moment is that there is no developed portable device for lower limb functional rehabilitation of patients with neurological disorders and stroke which could lead to new functional rehabilitation methods.

\section{GAIT ANALYSIS}

The mechanical development of any mobility-aid device is based in the biomechanical analysis of the human morphology and the human gait [10]. So these devices must consistent with the wearer on the ergonomic level, and on the other hand must be able to act with patients during the gait cycle. This includes not only mechanical design procedure but also on the mechanical sizing of the exoskeleton (Hip and Knee Torque/velocity, the required power, ...etc.) and the selection of drive module on each joint.

So as mentioned before, understanding the biomechanical data of a walking human is decisive in the design of active orthotics and exoskeletons for the lower extremities. Hence, defining the gait cycle as a cyclic process, which starts and ends with heel strike from the same leg. Generally it can be divided into 2 main phases: Stance and swing phase. Stance phase is the phase at which the body weight is supported by the concerned leg (right leg as shown in Fig. 2), while swing phase is when the leg is swinging to get ready for the next loading.

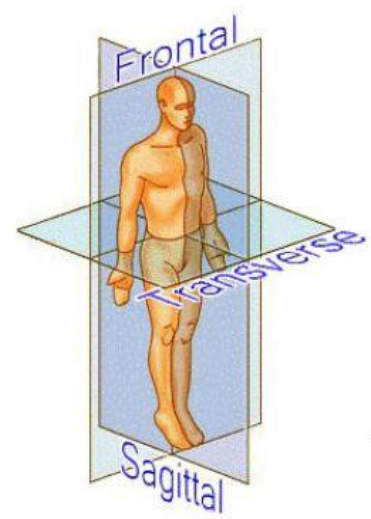

Fig. 1. Anatomical planes.

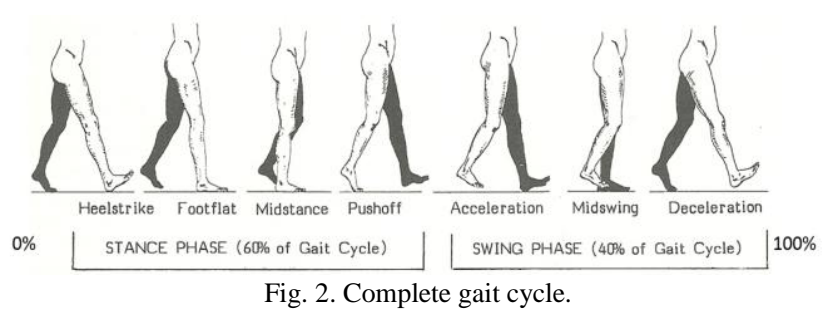

The normal human leg contains 7 DoF [8] and [11] three of them are for hip and one for knee and three for ankle, the toes are out of our scope; this enable humans doing complicated motions and actions which permits human body maneuverability while maintaining his stability.

Most of body muscles and bones are active and in motion during human walking procedure, in order to understand the body motions, one should take all anatomical planes in consideration see Fig. 1, but, it is found the dominant plane in studying the gait kinematics is the Sagittal plane.

At the start of gait cycle, the body is supported by the two legs (double supported), and the weight of the body is 
transferred from heel raised leg to the heel stroked leg. The shock is absorbed as the weight is transferred rapidly during the loading response period.

Consequently, body weight is transferred to the concerned leg, it is pushed by means of momentum forwardly and to the up direction, then, the concerned foot heel rise and the opposite foot strikes the ground. At this instant, the body is also double supported till the load is fully transferred to contralateral limb.

Swing phase is started with lifting the foot from the floor and ends when the foot is heel stroked to the ground. The ankle, hip, knee joints objectives are to keep a clearance with the ground and prepare the foot for the next heel strike.

Whilst the walking bio-mechanical data differs across subject, region, and conditions, but the qualitative kind of these data remains similar [13], in our design we take the data for $1.4 \mathrm{~km} / \mathrm{h}$ which is an average walking speed that could be useful in case of children. So by studying the hip and knee joints, and based on the bio-mechanical data extracted from 3 with respect walking linear speed for children, it is found the hip angle varies from range of $-20^{\circ}$ to $28^{\circ}$ while performing normal gait motion, and knee angle are ranging from $0^{\circ}$ to $64^{\circ}$ as shown in Fig. 3 .

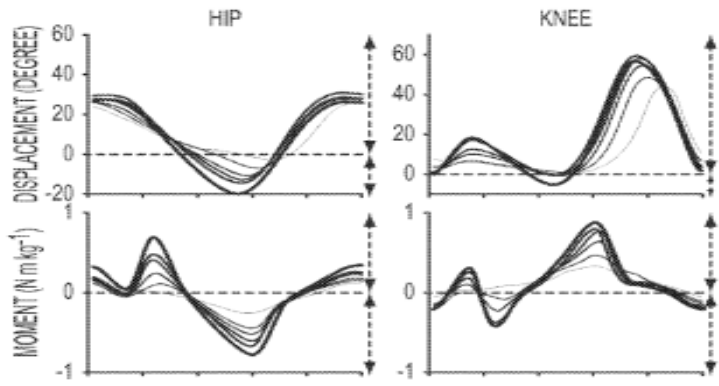

Fig. 3. Angle and torques for hip and knee joints with respect to gait cycle, Trajectories are for six walking speeds ranging from 1 to $1.6 \mathrm{Km} / \mathrm{h}$ [14].

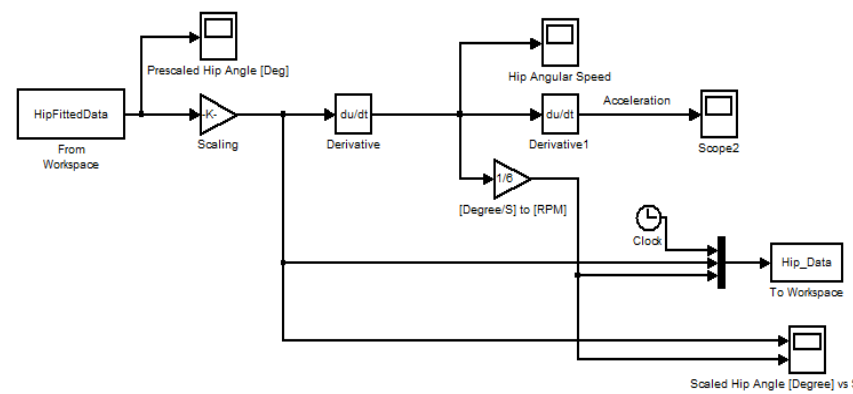

Fig. 4. Matlab-Simulink model to calculate hip motion curve derivatives.

In order to calculate the speed of Hip and knee joints, one should apply differentiation to the curves of angles with respect to time as shown in Eq. (1).

$$
\omega=\dot{\theta}=\frac{d \theta}{d t}
$$

For curve differentiation procedure, it is performed with aid of a simple Matlab-Simulink model as shown in Fig. 4, the data is fed to the model with 0.1 time step to be differentiated, then converting the data values from [deg/s] into [rpm], and finally the data is plotted and saved.

By combining the data of Hip and knee angle, Speed, and torque, the following charts, see Fig. 5, could be concluded.

Hip and Knee torque curves can be found normalized per unit mass as shown in Fig. 6.
From the charts indicated in Fig. 5, and Fig. 6, one can summarize the normal walking characteristics in following Table II.

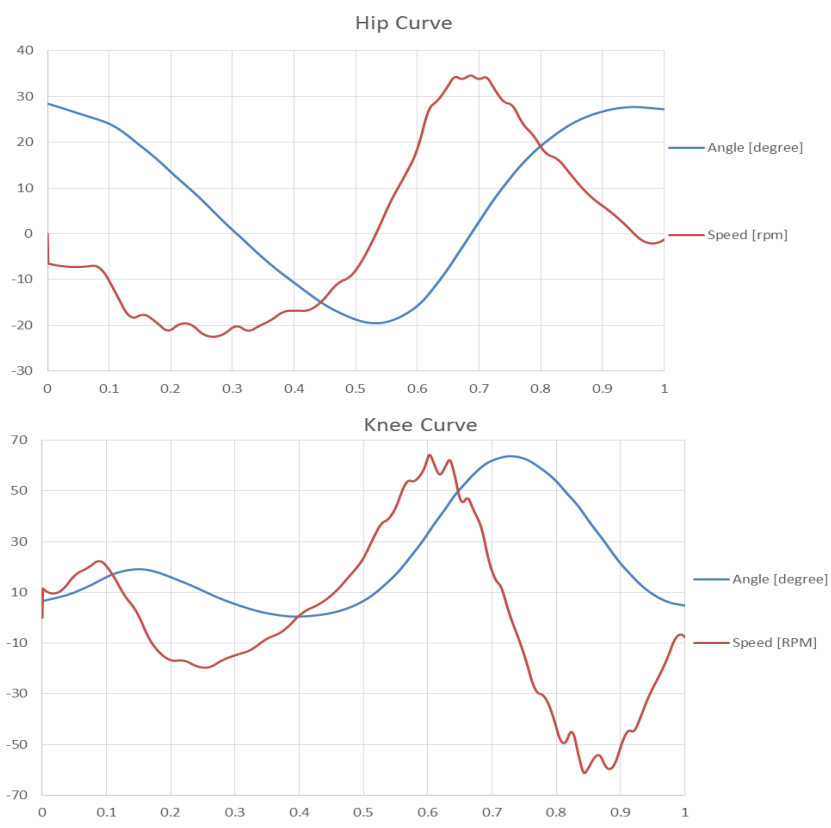

Fig. 5. Angles, and speeds for hip and knee curves w.r.t. gait cycle.
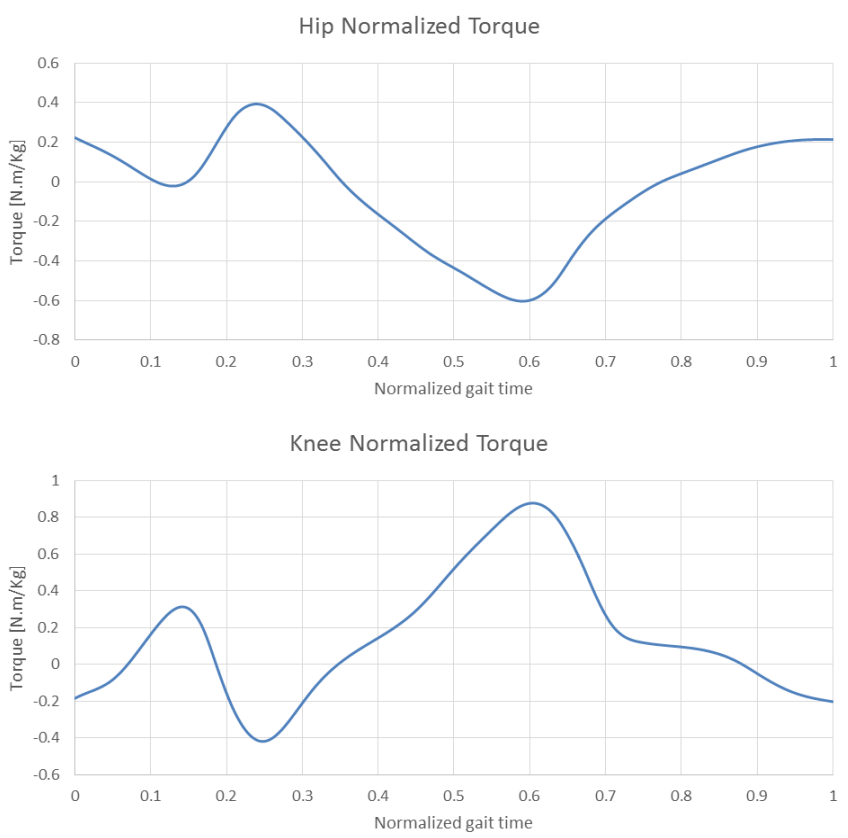

Fig. 6. Hip and knee normalized torques per unit mass w.r.t. gait cycle.

\section{A. Proposed System}

The system is proposed to be mechanically attached to patient's lower limbs by rubber belts. External balance system is used by the wearer to maintain stability, the device itself, in our first version, can be simplified into six moving degrees of two freedom in sagittal plane, two for hips (flexion/extension), for knees (flexion/extension), and tow for ankles (dorsal/plantar). On the other hand, the Hips and the knees are actuated by servo actuators, while the ankles will be driven using a springs which will help to return it to its neutral position during the swing phase, so using those springs can contribute in preventing dragging the foot. The system also includes a back pack, used to hold the power supply, controlling boards, motor drivers, and sensors to 
measure body motion.

TABLE II: NORMAL GAIT CHARACTERISTICS

\begin{tabular}{lccc}
\hline & Angle [deg] & Speed [rpm] & Torque [N.m/Kg] \\
\hline Hip joint & From-19 to 28 & 35 & 0.6 \\
\hline Knee joint & 0 to 64 & 63 & 0.88 \\
\hline
\end{tabular}

\section{B. System Parameters}

The proposed system parameters are listed in Table III. Body mass and lengths are got from a medical report and the others are approximated based on published ratios [15]. Some of medical data are multiplied by a safety factor $(\approx 6 \%)$, in order to increase system durability.

TABLE III: BODY AND SYSTEM PARAMETERS

\begin{tabular}{|c|c|c|}
\hline \multicolumn{3}{|l|}{ Masses } \\
\hline Mass of the whole Body & 50 & {$[\mathrm{Kg}]$} \\
\hline Mass of upper Trunk (with Head and Arms) & 29.115 & {$[\mathrm{Kg}]$} \\
\hline Mass of Backpack & 15 & {$[\mathrm{Kg}]$} \\
\hline Mass of upper Trunk + Backpack & 44.115 & {$[\mathrm{Kg}]$} \\
\hline Mass of Leg & 10.4425 & {$[\mathrm{Kg}]$} \\
\hline Mass of Thigh & 7.39 & {$[\mathrm{Kg}]$} \\
\hline Mass of Shank & 2.405 & {$[\mathrm{Kg}]$} \\
\hline Mass of foot & 0.6475 & {$[\mathrm{Kg}]$} \\
\hline System Mass at the HIP (per each) & 2.5 & {$[\mathrm{Kg}]$} \\
\hline System Mass at the Knee (per each) & 2.5 & {$[\mathrm{Kg}]$} \\
\hline System Mass at Thigh & 1 & {$[\mathrm{Kg}]$} \\
\hline System Mass at Shin & 1 & {$[\mathrm{Kg}]$} \\
\hline System Mass at Foot & 1.5 & {$[\mathrm{Kg}]$} \\
\hline Total System Mass for one Leg & 8.5 & {$[\mathrm{Kg}]$} \\
\hline Total System Mass & 32 & {$[\mathrm{Kg}]$} \\
\hline Total Body and System Masses & 82 & {$[\mathrm{Kg}]$} \\
\hline \multicolumn{3}{|l|}{$\begin{array}{l}\text { Lenghts } \\
\end{array}$} \\
\hline Total length of Body & 1.4 & {$[\mathrm{~m}]$} \\
\hline Trunk length & 0.63 & {$[\mathrm{~m}]$} \\
\hline Leg Length & 0.77 & {$[\mathrm{~m}]$} \\
\hline Thigh Length & 0.3465 & {$[\mathrm{~m}]$} \\
\hline Shin Length & 0.4235 & {$[\mathrm{~m}]$} \\
\hline
\end{tabular}

\section{ACTUATOR SIZING}

In order to estimate the power required for each joint, and select motor needed to drive the load, one should firstly calculate the load worst case conditions, i.e. maximum Torque needed, and maximum Speed also. These calculations are done in quasi-static conditions.

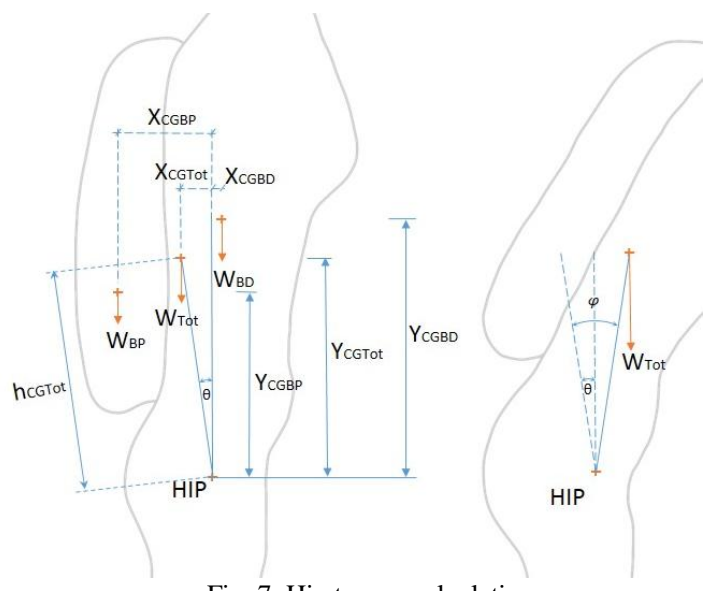

Fig. 7. Hip torque calculations.

\section{A. Hip Actuation Torque Calculations}

1) Requirement: During swing mode for a leg, the other supporting hip should be able to hold the upper trunk with its backpack bended up to $40^{\circ}$ to the front.

2) Assumptions:

- The Center of Gravity (CG) of the upper body is above the hip center of rotation by $55 \%$ of upper body height.

- $\mathrm{CG}$ is located at the center of the thickness of both body and backpack.

- Body thickness will be considered $0.2 \mathrm{~m}$ while backpack thickness is considered to be $0.15 \mathrm{~m}$.

3) Calculations: In order to calculate the magnitude and location of the CG for both of body and back-pack, see Fig. 7 :

a) Magnitude:

$$
\begin{array}{r}
W=m \times g \\
W_{T o t}=W_{B D}+W_{B P}
\end{array}
$$

b) Location:

And,

$$
\begin{gathered}
W_{B D}\left(X_{C G B D}+X_{C G T o t}\right)=W_{B P}\left(X_{C G B P}-X_{C G T o t}\right) \\
X_{C G T o t}=\frac{W_{B P} X_{C G B P}-W_{B D} X_{C G B D}}{W_{B P}+W_{B D}}
\end{gathered}
$$

$$
\begin{gathered}
W_{B D}\left(Y_{C G B D}-Y_{C G T o t}\right)=W_{B P}\left(Y_{C G T o t}-Y_{C G B P}\right) \\
Y_{C G T o t}=\frac{W_{B P} Y_{C G B P}-W_{B D} Y_{C G B D}}{W_{B P}+W_{B D}}
\end{gathered}
$$

To calculate the angle $\left(\theta_{\text {CGTot }}\right)$

$$
\theta_{\text {CGTot }}=\tan ^{-1}\left(\frac{X_{\text {CGTot }}}{Y_{\text {CGTot }}}\right)
$$

At stand, still situation, the torque can be calculated as:

$$
T_{H i p, \varphi=0^{\circ}}=W_{\text {Tot }} \times X_{C G T o t}
$$

In order to calculate the distance between hip and CG:

$$
h_{\text {CGTot }}=\sqrt{X_{\text {CGTot }}^{2}+Y^{2}{ }_{\text {CGTot }}}
$$

It is required to bend the upper trunk by $30^{\circ}$, so the torque will be:

$$
T_{\text {Hip }, \varphi \neq 0^{\circ}}=W_{\text {Tot }} \times h_{C G T o t} \times \sin (\varphi-\theta)
$$

Numerical application are illustrated in table IV.

\section{B. Knee actuation torque calculations}

1) Requirement:

- One knee should be able to support body weight while walking.

2) Assumptions:

- Max. Hip flexion angle is $10^{\circ}$ while for extension is $20^{\circ}$.

- The center of gravity for the leg is located at the knee.

- The right knee is considered to be fixed at ground.

3) Calculations: In order to calculate the required torque sufficient for the knee to perform normal gait, it required to perform the calculations in the worst case configuration, while the whole body is supported by one leg.

In case of shown in Fig. 8, the whole body is supported by the right leg, the right knee should counterbalance the 
weight of the entire body.

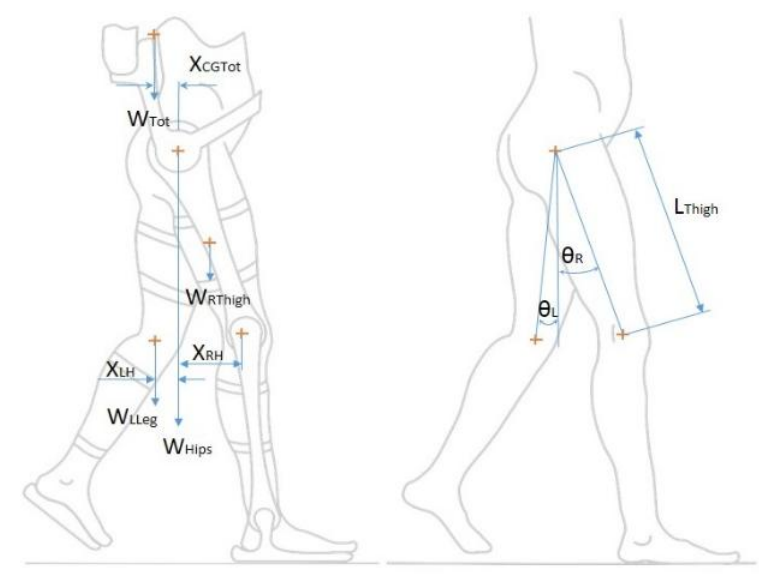

Fig. 8. Knee torque calculations during gait.

TABLE IV: TORQUE CALCULATIONS FOR HIP

\begin{tabular}{lccccc}
\hline \multicolumn{1}{c}{ Variable } & Value & Unit & Variable & Value & Unit \\
\hline Body Width & 0.2 & $m$ & $\varphi$ & 40 & deg \\
\hline $\begin{array}{l}\text { Backpack } \\
\text { width }\end{array}$ & 0.15 & $m$ & $W_{\text {Tot }}$ & 432.7682 & $N$ \\
\hline$W_{B D}$ & 285.6182 & $N$ & $X_{\text {CGTot }}$ & 0.054504 & $m$ \\
\hline$W_{B P}$ & 147.15 & $N$ & $Y_{\text {CGTot }}$ & 0.330689 & $m$ \\
\hline$X_{C G B D}$ & 0.005 & $m$ & $h_{\text {CGTot }}$ & 0.335151 & $m$ \\
\hline$X_{C G B P}$ & 0.17 & $m$ & $\theta_{\text {CGTot }}$ & 9.359244 & $\mathrm{deg}$ \\
\hline$Y_{C G B D}$ & 0.3465 & $m$ & $T_{\text {Hip } . \varphi=0}$ & 23.58741 & $\mathrm{~N} . \mathrm{m}$ \\
\hline$Y_{\text {CGBP }}$ & 0.3 & $m$ & $T_{\text {Hip . } \varphi=40}$ & 73.92142 & $\mathrm{~N} . m$ \\
\hline
\end{tabular}

Distance between the left (Unsupported leg) and the Hips center is considered $X_{L H}$, where the distance from the right (Supported leg) and the center of Hips is to be $X_{R H}$. In the current legs configuration, max. hip flexion angle will be note by $\theta_{L}$ while $\theta_{R}$ for extension.

$$
\begin{gathered}
X_{L H}=L_{\text {Thigh }} \sin \theta_{L} \\
X_{R H}=L_{\text {Thigh }} \sin \theta_{R}
\end{gathered}
$$

Torque affected by upper body and the backpack exerts at the upper body $\mathrm{CG}$, where the weight is $W_{C G T o t}$.

$$
\begin{gathered}
T_{\text {Tot } \varphi=0}=W_{\text {Tot }}\left(X_{C G T o t}+X_{R H}\right) \\
T_{\text {Tot } \varphi \neq 0}=W_{\text {Tot }}\left(X_{R H}-h_{C G T p t} \times \sin (\varphi-\theta)\right)
\end{gathered}
$$

Weight of both of Hips actuators $W_{\text {Hips }}$ and located at the center of Hips produce the following torque:

$$
T_{\text {Hips }}=W_{\text {Hips }} X_{R H}
$$

The weight of the unsupported leg with system attached is considered ( $W_{L L e g}$ ) and located at the left knee:

$$
T_{\text {LLeg }}=W_{\text {Leeg }}\left(X_{L H}+X_{R H}\right)
$$

Torque generated by the Weight of the thigh and the attached supporting link:

$$
T_{R T h i g h}=W_{R T h i g h} \frac{X_{R H}}{2}
$$

\begin{tabular}{|c|c|c|c|c|c|}
\hline Variable & Value & Unit & Variable & Value & Unit \\
\hline$\theta_{L}$ & 10 & deg & $X_{L H}$ & 0.060169 & $m$ \\
\hline$\theta_{R}$ & 20 & deg & $X_{R H}$ & 0.11851 & N.m \\
\hline$W_{T o t}$ & 432.7682 & $N$ & $T_{T o t \varphi}=0$ & 74.87475 & $N . m$ \\
\hline$X_{\text {CGTot }}$ & 0.054504 & $m$ & $T_{T o t \varphi \neq 0}$ & 37.031 & $N . m$ \\
\hline$W_{R T h i g h}$ & 82.3059 & $N$ & $T_{\text {Hips }}$ & 5.812915 & $N . m$ \\
\hline$W_{\text {LLeg }}$ & 161.3009 & $N$ & $T_{R T h i g h}$ & 4.877035 & $N . m$ \\
\hline$W_{\text {Hips }}$ & 49.05 & $N$ & $T_{L T h i g h}$ & 28.8211 & $N . m$ \\
\hline \multirow[t]{2}{*}{$L_{T h i g h}$} & 0.3465 & $m$ & $T_{\text {Knee. } \varphi=0}$ & 114.3858 & $N . m$ \\
\hline & & & $T_{\text {knee . } \varphi=10}$ & 89.17637 & $N . m$ \\
\hline
\end{tabular}

Summing the results of equations (11), (12), (13), and (14) this leads to:

$$
T_{\text {Knee }}=T_{\text {Tot }}+T_{\text {Hips }}+T_{\text {LLeg }}+T_{\text {RThigh }}
$$

And by applying numerical values, one will find Table $\mathrm{V}$.

TABLE V: TORQUE CALCULATIONS FOR KNEE

\section{Hip Speed Calculation}

Considering the walking speed is to be 1 complete cycle (from $0 \%$ to $100 \%$ as shown in Fig. 2) in 1 second which is equivalent to the required walking speed $1.4 \mathrm{Km} / \mathrm{h}$.

As shown in Fig. 5, the max. Hip angle is $35^{\circ}$, this for healthy adult man, but in objected case; the proposed exoskeleton is designed to have max. Hip motion angle is to be $20^{\circ}$, so this data should be scaled before applying differentiation process.

From Fig. 9, the max speed is indicated as approximately $21[\mathrm{rpm}]$ and this occurs at instant 0.7 [S].
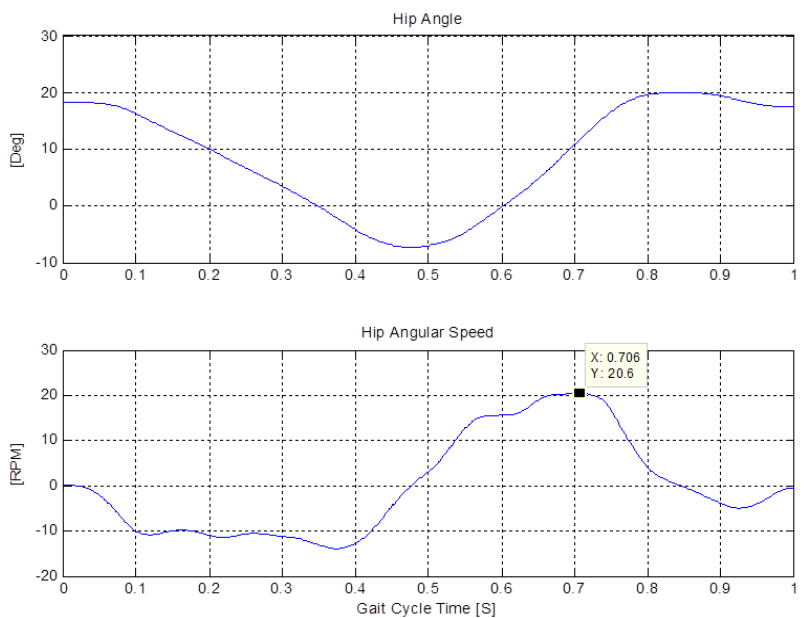

Fig. 9. Hip degree of motion curve.

\section{Knee Speed Calculation}

Also, to get Speed curve for knee joint, scaling and differentiation processes with respect to time have to be carried out to the knee motion curve.

From Fig. 10, it's obvious that the max. Speed required for knee joint is approximately 34 [rpm], and that occurs when gait time cycle reaches at 0.5 [S].

\section{RESULTS}

From previous calculations, one can summarize the following results:

From table VI, it's obvious that knee joint actuator seems to be more powerful than the hip joint, but in order to standardize the proposed exoskeleton, one actuator can be 
fitted to the both joints.

The suggested actuator should satisfy the joints requirements. It be capable to overcome 130 N.m torque, and should have angular speed not less than of 35 [rpm].
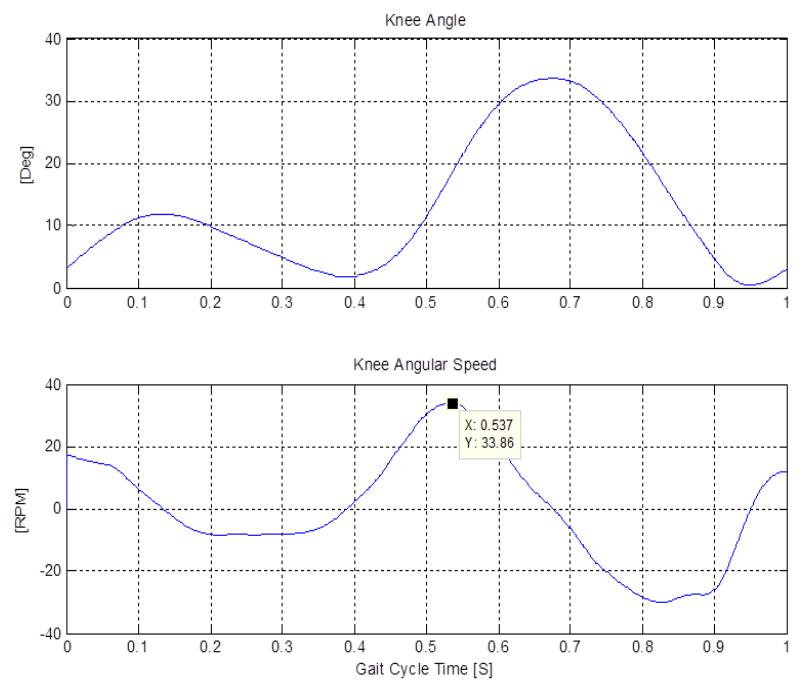

Fig. 10. Knee Degree of Motion curve.

\section{CAlCUlations VerificATION}

In order to test and validate the achieved results, a virtual model is constructed using Matlab-Simulink. This model shown in Fig. 11 is constructed of imported 3d drawings from Catia, the imported parts have their mechanical parameters, like weights and CG location, so the model should simulate the weight distribution of the real exoskeleton.

The model is used to verify the torque calculations, so, same prescribed poses were applied to the model, and both of hip and knee torques were measured and verified.

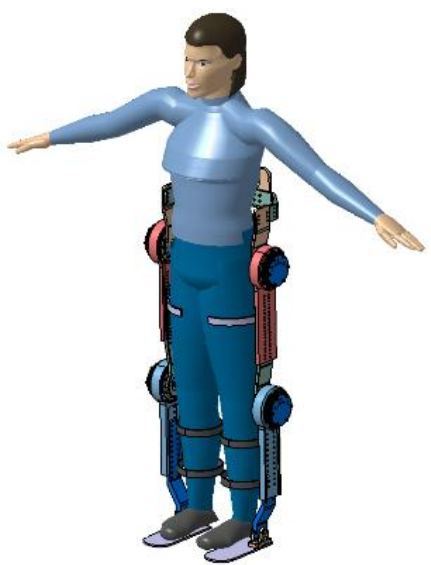

Fig. 11. Exoskeleton model.

TABLE VI: MAX SPEED AND TORQUE FOR HIP AND KNEE JOINTS

\begin{tabular}{lcccc}
\hline \multirow{2}{*}{$\begin{array}{l}\text { Joint } \\
\text { actuation }\end{array}$} & \multicolumn{3}{c}{ Required Torque [N.m] } & $\begin{array}{c}\text { Max. } \\
\text { Speed } \\
{[\mathrm{rpm}]}\end{array}$ \\
\cline { 2 - 5 } Hip & $\begin{array}{c}0.6 \times 82 \\
=49.2\end{array}$ & 51.128 & $\approx 104 \%$ & 21 \\
\hline Knee & $\begin{array}{l}0.8 \times 82 \\
=72.16\end{array}$ & 123.58 & $\approx 173 \%$ & 34 \\
\hline
\end{tabular}

Thus, choosing 2 active joints per leg will satisfy simplicity criteria as well as reduce the overall weight, also allowing the future exoskeleton to be affordable in price.
The worst case poses are assumed during the gait cycle, and torque calculations are applied with respect to body dimensions, as it was safe compared to normalized biomechanical charts as illustrated. Actuator speeds, are calculated by differentiating the joint trajectories. Worst combinations, are selected to be the requirements for the actuator. This is due to match the modularity in design as well as easy maintenance requirements.

Finally, the testing and validation process is carried out using a virtual model of the patient wearing exoskeleton, performing a normal gait. Results show that the actuation parameters selected are safe enough to perform the gait cycle by a suitable factor.

\section{FUTURE WORK}

Future works regarding the development of the exoskeleton will focuses on several domains: around establishing the advanced technologies like accumulators, lightweight servo-actuators, and efficient transmissions. There are a few points related to the mechanical design of exoskeleton, the understanding of muscle behavior during walking and other tasks in order to lead to an effective mechanical architectures of exoskeleton. Interfacing the exoskeleton to the patients on both mechanical and control domains will be treated in the future work.

Also, the device wearer's safety should be studied since the patients are wearing powerful devices and they couldn't counter act any malfunctions of the exoskeleton.

\section{ACKNOWLEDGMENT}

The authors of this paper would like to give thanks to Sandyc industries for their notable funding, supports and efforts in this research project.

\section{REFERENCES}

[1] H. Kawamoto and Y. Sankai, "Comfortable power assist control method for walking aid by hal-3," in Proc. IEEE International Conference on Systems, Man and Cybernetics, 2002, vol. 4, p. 6

[2] A. M. Technologies. (2016). New rewalk personal 6.0 - rewalk more than walking. [Online]. Available: www.rewalk.com

[3] L. Mertz, "The next generation of exoskeletons: Lighter, cheaper devices are in the works," IEEE Pulse, vol. 3, no. 4, pp. 56-61, 2012.

[4] R. J. Farris, "Design of a powered lower-limb exoskeleton and control for gait assistance in paraplegics," Thesis, 2012.

[5] SuitX. (2016). Phoenix exoskeleton. [Online]. Available: http://www.suitx.com/phoenix

[6] A. B. Zoss, H. Kazerooni, and A. Chu, "Biomechanical design of the berkeley lower extremity exoskeleton (bleex)," IEEE/ASME Transactions on Mechatronics, vol. 11, no. 2, pp. 128-138, 2006.

[7] G. Zeilig, H. Weingarden, M. Zwecker, I. Dudkiewicz, A. Bloch, and A. Esquenazi, "Safety and tolerance of the rewalk() exoskeleton suit for ambulation by people with complete spinal cord injury: A pilot study," The Journal of Spinal Cord Medicine, vol. 35, no. 2, pp. 96$101,2012$.

[8] A. Zoss, H. Kazerooni, and A. Chu, "On the mechanical design of the berkeley lower extremity exoskeleton (bleex)," in Proc. 2005 IEEE/RSJ International Conference on Intelligent Robots and Systems, pp. 3465-3472.

[9] S. Alfayad, F. B. Ouezdou, F. Namoun, and G. Cheng, "High performance integrated electro-hydraulic actuator for robotics. part ii: Theoretical modelling, simulation, control \& comparison with rea measurements," Elsevier, vol. 169, no. 1, pp. 124-132, 2011.

[10] H. Zabaleta, M. Bureau, G. Eizmendi, E. Olaiz, J. Medina, and M. Perez, "Exoskeleton design for functional rehabilitation in patients with neurological disorders and stroke," in Proc. 2007 IEEE 10th International Conference on Rehabilitation Robotics, June 2007, pp. 112-118. 
[11] S. Alfayad, F. B. Ouezdou, F. Namoun, O. Bruneau, and P. Henaff, "Three dof hybrid mechanism for humanoid robotic application: Modeling, design and realization," in Proc. 2009 IEEE/RS International Conference on Intelligent Robots and Systems, 2009, pp. 4955-4961.

[12] R. Schafer. (1987). Clinical Biomechanics: Musculoskeletal Actions and Reactions. Williams \& Wilkins. [Online]. Available: https://books.google.fr/books?id=iChtAAAAMAAJ

[13] A. J. Van Den Bogert, "Exotendons for assistance of human locomotion," Biomed. Eng.Online, vol. 2, no. 2, p. 17, 2003.

[14] O. Unluhisarcikli, C. Mavroidis, P. Bonato, M. Pietrusisnki, and B. Weinberg, "Lower extremity exoskeleton for gait retraining," 2013.

[15] P. de Leva, "Adjustments to zatsiorsky-seluyanov's segment inertia parameters," J. Biomech, vol. 29, no. 9, pp. 1223-1230, 1996.

Kardofaki Mohamad received his BEng. and MEng. in mechatronics engineering from the Faculty of engineering of the Lebanese University, Lebanon in 2014. He is now a PhD student at Paris-Saclay University. His research interests include active exoskeletons, humanoid robots, electric and hydraulic actuators and mobile robot manipulators.

Moustafa A. Fouz received the B.Sc. degree from Arab Academy for Science, Technology and Maritime Transport (AASTMT), Egypt in 2009. $\mathrm{He}$ has got his MSc also from AASTMT (Alexandria Branch) in 2013. He is a teacher assistant of mechatronics program at Mechanical Engineering Department in AASTMT (Cairo Branch). Currently, he is in a PhD scholarship to LISV at UVSQ in France. His interests include robotic systems and mechatronics applications.
Khaled Fouda received his BSc. and MSc. in computer engineering from Cairo University, Egypt, and Arab Academy for Science \& Technology \& Maritime Transport, Egypt in 2004 and 2014, respectively. Since 2005, he worked at IBM in many positions including Project Manager, Regional Consultant, then he became Managing Director, and founder for FodaDev Co., Ltd, from 2007 to 2011 he was also lecturer at School of Business, Cape Breton University, and he is now PhD student at UVSQ. His research interests include humanoid robot, and service robot.

Samer AlFayad received his master diploma in sciences and technology from Ecole Nationale Supérieure d'Arts et Métiers (ENSAM-Paris) in 2005. His Ph.D was received in robotic development from Versailles University (UVSQ) in 2009. He was awarded of the best Ph.D. thesis in robotics for year 2010 by the French CNRS. Also, he was awarded of the best Ph.D. thesis in robotics for the 20 years' anniversary of UVSQ. From 2010 to 2011 he was a post-doc researcher at Technische Universitat Munich (TUM-Germany) with a scholarship from the Alexander Von Humboldt foundation. In 2011, he has been appointed as an associated professor in Humanoid robotic design at Versailles University. From 2012, he holds an industrial excellence chair about hydraulic domestication at UVSQ. He has been investigator in several French National projects. He is the leader of the Humanoid research group at LISV laboratory. He is working currently on the two anthropomorphic biped robots called HYDROiD and ROMEO2 at the LISV. He was the team leader of Paris-Saclay team to Mohamed Bin Zayed International Robotics Challenge MBZIRC2017. He is cocoordinator of Institute for Control and Decision (iCode Paris-Saclay). 